\title{
Neutrino masses and mixing from flavour antisymmetry
}

\author{
Anjan S. Joshipura \\ Physical Research Laboratory, \\ Navarangpura, Ahmedabad, 380009, India \\ E-mail: anjan@prl.res.in
}

ABSTRACT: We discuss consequences of assuming $(i)$ that the (Majorana) neutrino mass matrix $M_{\nu}$ displays flavour antisymmetry, $S_{\nu}^{T} M_{\nu} S_{\nu}=-M_{\nu}$ with respect to some discrete symmetry $S_{\nu}$ contained in $\mathrm{SU}(3)$ and $(i i) S_{\nu}$ together with a symmetry $T_{l}$ of the Hermitian combination $M_{l} M_{l}^{\dagger}$ of the charged lepton mass matrix forms a finite discrete subgroup $G_{f}$ of $\mathrm{SU}(3)$ whose breaking generates these symmetries. Assumption $(i)$ leads to at least one massless neutrino and allows only four textures for the neutrino mass matrix in a basis with a diagonal $S_{\nu}$ if it is assumed that the other two neutrinos are massive. Two of these textures contain a degenerate pair of neutrinos. Assumption (ii) can be used to determine the neutrino mixing patterns. We work out these patterns for two major group series $\Delta\left(3 N^{2}\right)$ and $\Delta\left(6 N^{2}\right)$ as $G_{f}$. It is found that all $\Delta\left(6 N^{2}\right)$ and $\Delta\left(3 N^{2}\right)$ groups with even $N$ contain some elements which can provide appropriate $S_{\nu}$. Mixing patterns can be determined analytically for these groups and it is found that only one of the four allowed neutrino mass textures is consistent with the observed values of the mixing angles $\theta_{13}$ and $\theta_{23}$. This texture corresponds to one massless and a degenerate pair of neutrinos which can provide the solar pair in the presence of some perturbations. The well-known groups $A_{4}$ and $S_{4}$ provide examples of the groups in respective series allowing correct $\theta_{13}$ and $\theta_{23}$. An explicit example based on $A_{4}$ and displaying a massless and two quasi degenerate neutrinos is discussed.

Keywords: Neutrino Physics, Discrete and Finite Symmetries

ArXiv EPrint: 1506.00455 


\section{Contents}

1 Introduction $\quad 1$

2 Allowed textures for neutrino mass matrix 2

3 Group theoretical determination of mixing 4

$3.1 \Delta\left(3 N^{2}\right) \quad 5$

$\begin{array}{ll}3.2 \Delta\left(6 N^{2}\right) & 9\end{array}$

$\begin{array}{lll}3.2 .1 & \text { Texture I } & 10\end{array}$

$\begin{array}{lll}3.2 .2 & \text { Texture IV } & 13\end{array}$

4 More predictive scenario: $Z_{2} \times Z_{2}$ symmetry 14

5 An $A_{4}$ model with flavour antisymmetry $\quad 15$

$\begin{array}{llr}6 & \text { Summary } & 18\end{array}$

\section{Introduction}

Orderly pattern of neutrino mixing appears to hide some symmetry, discrete or continuous. It is possible to connect a given mixing pattern with some discrete symmetries of the leptonic mass matrices. Such symmetries may however be residual symmetries arising from a bigger symmetry in the underlying theory. One can obtain a possible larger picture by assuming that these symmetries are a part of a bigger group operating at the fundamental level whose breaking leads to the symmetries of the mass matrices. There is an extensive literature on study of possible residual symmetries of the mass matrices and of the groups which harbor them [1-16], see [17-19] for reviews and additional references.

Starting point in these approaches is to assume the existence of some symmetries $S_{\nu}$ (usually a $Z_{2} \times Z_{2}$ ) and $T_{l}$ (usually $Z_{N}, N \geq 3$ ) of the (Majorana) neutrino and the charged lepton mass matrices

$$
\begin{gathered}
T_{l}^{\dagger} M_{l} M_{l}^{\dagger} T_{l}=M_{l} M_{l}^{\dagger}, \\
S_{\nu}^{T} M_{\nu} S_{\nu}=M_{\nu}
\end{gathered}
$$

Matrices diagonalizing the $3 \times 3$ symmetry matrices $S_{\nu}, T_{l}$ can be related to the mixing matrices in each sector. The structures of these matrices can also be independently fixed if one assume that $S_{\nu}$ and $T_{l}$ represent specific elements of some discrete group $G_{f}$ in a given three dimensional representation. In this way, the leptonic mixing can be directly related to group theoretical structures. This reasoning has been used for the determination of the 
neutrino mixing angles in case of the three non-degenerate neutrinos [1-15, 20], two or three degenerate neutrinos [21, 22] and one massless and two non-degenerate neutrinos [23, 24].

The residual symmetries may arise from the spontaneous breaking of $G_{f}$ if the vacuum expectation values of the Higgs fields responsible for generating neutrino (the charged lepton) masses break $G_{f}$ but respect $S_{\nu}\left(T_{l}\right)$. We wish to study in this paper consequences of an alternative assumption that the spontaneous breaking of $G_{f}$ leads to an $M_{\nu}$ which displays antisymmetry instead of symmetry, i.e. assume that eq. (1.2) gets replaced by

$$
S_{\nu}^{T} M_{\nu} S_{\nu}=-M_{\nu}
$$

but eq. (1.1) remains as it is. These assumptions prove to be quite powerful and are able to simultaneously restrict both the mass patterns and mixing angles when embedding of $S_{\nu}, T_{l}$ into $G_{f}$ is considered. In the case of symmetry, the vacuum determining neutrino masses is invariant under $S_{\nu}$. This invariance implies eq. (1.2). In the case of eq. (1.3), the vacuum is not invariant under $S_{\nu}$ but transforms under it in such a way that the neutrino mass matrix displays a residual antisymmetry. We shall give an explicit example based on $A_{4}$ of this phenomena in section 5 . Since $S_{\nu}$ in the antisymmetric case does not survive as a residual symmetry of $G_{f}$, eq. (1.3) and the requirement that $S_{\nu}$ belongs to $G_{f}$ are logically independent requirements. But they both can be consistent. Assuming that $S_{\nu}$ belongs to $G_{f}$ helps in determining the mixing pattern and we shall make this assumption. We shall further assume that $G_{f}$ is some finite discrete subgroup of $\mathrm{SU}(3)$. Then the first consequence of imposing eq. (1.3) is that Det $M_{\nu}=0$, i.e. at least one of the neutrinos remains massless. Since cases with two (or three!) massless neutrinos are not phenomenologically interesting, we shall restrict ourselves to cases with only one massless neutrino. Then as a second consequence of eq. (1.3), one can determine all the allowed forms of $M_{\nu}$ in a given basis for all possible $S_{\nu}$ contained in $\mathrm{SU}(3)$. There exist only four possible $M_{\nu}$ (and their permutations) consistent with eq. (1.3) in a particular basis with a diagonal $S_{\nu}$. Two of these give one massless and two non-degenerate neutrinos and the other two give a massless and a degenerate pair of neutrinos which may be identified with the solar pair.

We determine all the allowed textures of the neutrino mass matrix in the next section. Subsequently, we discuss groups $\Delta\left(3 N^{2}\right)$ and $\Delta\left(6 N^{2}\right)$ and identify those which can give correct description of mixing using flavour antisymmetry. In section 4, we introduce $Z_{2} \times Z_{2}$ as neutrino residual symmetry and present an example in which neutrino mass matrix gets fully determined group theoretically except for an overall scale. We discuss a realization of the basic idea with a simple example based on the $A_{4}$ group in section 5 . Section 6 contains summary and comparison with earlier relevant works.

\section{Allowed textures for neutrino mass matrix}

We shall first consider the case of only one $S_{\nu}$ satisfying eq. (1.3) and subsequently generalize it to include two. The unitary matrix $S_{\nu}$ can be diagonalized by another unitary matrix $V_{S_{\nu}}$ :

$$
V_{S_{\nu}}^{\dagger} S_{\nu} V_{S_{\nu}}=\tilde{S}_{\nu}
$$


where $\tilde{S}_{\nu}$ is a diagonal matrix having the form:

$$
\tilde{S}_{\nu}=\operatorname{diag} .\left(\lambda_{1}, \lambda_{2}, \lambda_{3}\right) .
$$

Unitarity of $S_{\nu}$ implies that $\lambda_{1,2,3}$ are some roots of unity. They satisfy $\lambda_{1} \lambda_{2} \lambda_{3}=1$ due to the condition Det $S_{\nu}=+1$. We now go to the basis with a diagonal $S_{\nu}$. Defining $\tilde{M}_{\nu}=V_{S_{\nu}}^{T} M_{\nu} V_{S_{\nu}}$, eq. (1.3) can be rewritten as:

$$
\left(\tilde{M}_{\nu}\right)_{i j}\left(1+\lambda_{i} \lambda_{j}\right)=0 \quad(i, j \text { not summed }) .
$$

It follows that a given element $\left(\tilde{M}_{\nu}\right)_{i j}$ is non-zero only if the factor in bracket multiplying it is zero. This cannot happen for an arbitrary set of $\lambda_{i}$ and one needs to impose specific relation among them to obtain a non-trivial $\tilde{M}_{\nu}$. We now argue that only two possible forms of $\tilde{S}_{\nu}$ and their permutations lead to neutrino mass matrices with two massive neutrinos. The third mass will always be zero as a consequence of eq. (1.3) and the assumption that $S_{\nu}$ belongs to $\mathrm{SU}(3)$. These forms of $\tilde{S}_{\nu}$ are given by:

$$
\begin{aligned}
& \tilde{S}_{1 \nu}=\operatorname{diag} .\left(\lambda,-\lambda^{*},-1\right), \\
& \tilde{S}_{2 \nu}=\operatorname{diag} .( \pm i, \mp i, 1) .
\end{aligned}
$$

$\lambda$ is an arbitrary root of unity. This can be argued as follows. Assume that at least one off-diagonal element of $\tilde{M}_{\nu}$ is non-zero which we take as the 12 element for definiteness. In this case, eq. (2.2) immediately implies the first of eq. (2.3) as a necessary condition. One can distinguish three separate cases of this condition $^{1}$ (I) $\lambda=1$ (II) $\lambda= \pm i$ and (III) $\lambda \neq \pm 1, \pm i$. The structures of $\tilde{M}_{\nu}$ get determined in these cases from condition eq. (2.2) as follows:

$$
\text { Texture I: } \quad \tilde{S}_{1 \nu}=(1,-1,-1) ; \quad \tilde{M}_{\nu}=m_{0}\left(\begin{array}{ccc}
0 & c & s e^{i \beta} \\
c & 0 & 0 \\
s e^{i \beta} & 0 & 0
\end{array}\right),
$$

where $c=\cos \theta, s=\sin \theta$. This structure implies one massless and two degenerate neutrinos with a mass $\left|m_{0}\right|$. In case of (II),

$$
\text { Texture II: } \quad \tilde{S}_{1 \nu}=( \pm i, \pm i,-1) ; \quad \tilde{M}_{\nu}=\left(\begin{array}{ccc}
x_{1} & y & 0 \\
y & x_{2} & 0 \\
0 & 0 & 0
\end{array}\right)
$$

This case corresponds to one massless and two non-degenerate neutrinos. In the third case one gets

$$
\text { Texture III: } \quad \tilde{S}_{1_{\nu}}=\left(\lambda,-\lambda^{*},-1\right) ; \quad \tilde{M}_{\nu}=m_{0}\left(\begin{array}{ccc}
0 & 1 & 0 \\
1 & 0 & 0 \\
0 & 0 & 0
\end{array}\right), \quad(\lambda \neq \pm 1, \pm i)
$$

which implies a massless and a pair of degenerate neutrinos.

\footnotetext{
${ }^{1} \lambda=-1$ case corresponds to permutation of the case with $\lambda=1$.
} 
The cases (I, III) lead to the same mass spectrum but different mixing patterns. $\tilde{M}_{\nu}$ in eq. (2.4) is diagonalized as $V_{\nu}^{T} \tilde{M}_{\nu} V_{\nu}=\operatorname{diag} .\left(m_{0}, m_{0}, 0\right)$ with

$$
V_{\nu}=\left(\begin{array}{ccc}
\frac{1}{\sqrt{2}} & -\frac{i}{\sqrt{2}} & 0 \\
\frac{c}{\sqrt{2}} & \frac{i c}{\sqrt{2}} & -s \\
\frac{s}{\sqrt{2}} e^{-i \beta} & \frac{i s}{\sqrt{2}} e^{-i \beta} & c e^{-i \beta}
\end{array}\right)\left(\begin{array}{ccc}
\cos \phi & -\sin \phi & 0 \\
\sin \phi & \cos \phi & 0 \\
0 & 0 & 1
\end{array}\right)
$$

The arbitrary rotation by an angle $\phi$ originates due to degeneracy in masses. The texture II in eq. (2.5) is diagonalized by a unitary rotation in the 12 plane while the one in eq. (2.6) by a similar matrix with the angle $\frac{\pi}{4}$.

The permutations of entries in $\tilde{M}_{\nu}$ give equivalent structures and are obtained by permuting entries in $\tilde{S}_{1 \nu}$. The case which is not equivalent to above textures follows with a starting assumption that one of the diagonal elements of $\tilde{M}_{\nu} \neq 0$ say, $\left(\tilde{M}_{\nu}\right)_{11} \neq 0$. In this case one requires $\tilde{S}_{\nu}=$ diag. $\left( \pm i, \lambda^{\prime}, \mp i \lambda^{* \prime}\right)$ with $\left|\lambda^{\prime}\right|=1$. The case with $\lambda^{\prime}= \pm i$ gives $\tilde{S}_{1 \nu}$ which is already covered. $\lambda^{\prime}=\mp i$ implies the condition $\tilde{S}_{2 \nu}$ in (2.3). This leads to a new texture

$$
\text { Texture IV: } \quad \tilde{S}_{\nu}=(i,-i, 1) ; \quad \tilde{M}_{\nu}=\left(\begin{array}{ccc}
x_{1} & 0 & 0 \\
0 & x_{2} & 0 \\
0 & 0 & 0
\end{array}\right) .
$$

For $\lambda^{\prime}= \pm 1$ one gets permutation of $\tilde{S}_{1 \nu}$ or $\tilde{S}_{2 \nu}$ and for $\lambda^{\prime} \neq \pm 1, \pm i$ only 11 element of $\tilde{M}_{\nu}$ is non zero and two neutrinos remain massless. Thus conditions eq. (2.3) and their permutations exhaust all possible textures of $\tilde{M}_{\nu}$ consistent with the antisymmetry of $M_{\nu}$, eq. (1.3) and two massive neutrinos. Any $G_{f}$ admitting an element with these sets of eigenvalues will give a viable choice for flavour antisymmetry group. Note that texture III (IV) can be obtained from I(II) by putting $s(y)$ to zero. But the residual symmetries in all four cases are different. Because of this, the embedding groups $G_{f}$ can also be different. We therefore discuss all these cases separately.

The mixing matrix in texture I contains two unknowns $\theta$ and $\beta$ apart from an overall complex scale $m_{0}$. This is a reflection of the fact that the corresponding $S_{\nu}$ is a $Z_{2}$ symmetry and contains two degenerate eigenvalues -1 . These unknowns can be fixed by imposing another residual $Z_{2}$ symmetry commuting with $S_{\nu}$ and satisfying eq. (1.2) or (1.3). We shall discuss such choices in section 4 .

\section{Group theoretical determination of mixing}

The physical neutrino mixing matrix $U_{\mathrm{PMNS}} \equiv U$ depends on the structure of $M_{\nu}$ and $M_{l} M_{l}^{\dagger}$. The latter can be determined if the symmetry $T_{l}$ as in eq. (1.1) is known. We now make an assumption that $S_{\nu}$ satisfying eq. (1.3) and $T_{l}$ as in eq. (1.1) are elements of some discrete subgroup (DSG) of SU(3) denoted by $G_{f}$. The DSG of SU(3) have been classified in [25-27]. They are further studied in [28-38]. These can be written in terms of few $3 \times 3$ presentation matrices whose multiple products generate various DSG. Two main groups series called $C$ and $D$ [37] constitute bulk of the DSG of SU(3). Of these, we shall explicitly study two infinite groups series $\Delta\left(3 N^{2}\right)$ and $\Delta\left(6 N^{2}\right)$ which are examples 
of the type $C$ and $D$ respectively. See [39-41] for earlier studies of neutrino mixing using the groups $\Delta\left(3 N^{2}\right)$ and $\Delta\left(6 N^{2}\right)$ and neutrino symmetry rather than antisymmetry.

Eq. (1.1) implies that $T_{l}$ commutes with $M_{l} M_{l}^{\dagger}$. Thus, the matrix $U_{l}$ diagonalizing the former also diagonalizes $M_{l} M_{l}^{\dagger}$ and corresponds to the mixing matrix among the left handed charged leptons. Similarly, the matrix $U_{\nu}$ diagonalizing $M_{\nu}$ gets related to the structure of $S_{\nu}$. In this way, the knowledge of $S_{\nu}$ and $T_{l}$ can be used to determine the mixing matrix

$$
U \equiv U_{\mathrm{PMNS}}=U_{l}^{\dagger} U_{\nu}
$$

This is the strategy followed in the general approach and we shall also use this to determine all possible mixing pattern for a given $G_{f}$ consistent with eqs. (1.1) and (1.3).

Not all the groups $G_{f}$ can admit an $S_{\nu}$ which will provide a legitimate antisymmetry operator $S_{\nu}$, i.e. an element with eigenvalues specified by eq. (2.3). Our strategy would be to determine a class of groups which will have one or more allowed $S_{\nu}$ and then look for all viable $T_{l}$ within these groups. There would be different mixing patterns associated with each choice of $S_{\nu}, T_{l}$ and it is possible to determine all of them analytically for $\Delta\left(3 N^{2}\right)$ and $\Delta\left(6 N^{2}\right)$ groups.

\section{$3.1 \Delta\left(3 N^{2}\right)$}

The $\Delta\left(3 N^{2}\right)$ groups are isomorphic to $\left(Z_{N} \times Z_{N}\right) \rtimes Z_{3}$, where $\rtimes$ denotes the semi-direct product. The group theoretical details for $\Delta\left(3 N^{2}\right)$ are discussed in [29, 42]. For our purpose, it is sufficient to note that all the elements of the group are generated from the multiple product of two basic generators defined as:

$$
F=\left(\begin{array}{ccc}
1 & 0 & 0 \\
0 & \eta & 0 \\
0 & 0 & \eta^{*}
\end{array}\right), \quad E=\left(\begin{array}{ccc}
0 & 1 & 0 \\
0 & 0 & 1 \\
1 & 0 & 0
\end{array}\right)
$$

with $\eta=e^{\frac{2 \pi i}{N}}$. Here $F$ generates one of the $Z_{N}$ groups and $E$ generates $Z_{3}$ in the semi-direct product $\left(Z_{N} \times Z_{N}\right) \rtimes Z_{3}$. The other $Z_{N}$ group is generated by $E F E^{-1}$. The above explicit matrices provide a faithful three dimensional irreducible representation of the group and multiple products of these matrices therefore generate the entire group whose elements can be labeled as:

$$
\begin{aligned}
W & \equiv W(N, p, q)=\left(\begin{array}{ccc}
\eta^{p} & 0 & 0 \\
0 & \eta^{q} & 0 \\
0 & 0 & \eta^{-p-q}
\end{array}\right), \quad R \equiv R(N, p, q)=\left(\begin{array}{ccc}
0 & 0 & \eta^{p} \\
\eta^{q} & 0 & 0 \\
0 & \eta^{-p-q} & 0
\end{array}\right) \\
V & \equiv V(N, p, q)=\left(\begin{array}{ccc}
0 & \eta^{p} & 0 \\
0 & 0 & \eta^{q} \\
\eta^{-p-q} & 0 & 0
\end{array}\right)
\end{aligned}
$$

All elements of $\Delta\left(3 N^{2}\right)$ are obtained by varying $p, q$ over the allowed range $p, q=0,1,2, \ldots$, $N-1$ in the above equation. Thus each matrices $W, R, V$ have $N^{2}$ elements giving in total $3 N^{2}$ elements corresponding to the order of $\Delta\left(3 N^{2}\right)$. The eigenvalue equation for the $2 N^{2}$ 
non-diagonal elements $R$ and $V$ is simply given by $\lambda^{3}=1$. These elements therefore have eigenvalues $\left(1, \omega, \omega^{2}\right)$ with $\omega=e^{\frac{2 \pi i}{3}}$. These are not in the form of eq. (2.3) required to get the neutrino antisymmetry operator $S_{\nu}$. Thus $S_{\nu}$ has to come from the $N^{2}$ diagonal elements. This requires that $N, p, q$ should be such that $W(N, p, q)=\operatorname{diag} .\left(\eta^{p}, \eta^{q}, \eta^{-p-q}\right)$ matches the required eigenvalues $\tilde{S}_{\nu}$ of $S_{\nu}$ given by eq. (2.3) or their permutations. This cannot happen for all the values of variables and one can easily identify the viable cases. It is found that

- $W$ can match any of $\tilde{S}_{\nu}$ only for even $N$. Thus only $\Delta\left(12 k^{2}\right)$ groups with $k=1,2, \ldots$ contain neutrino antisymmetry operator $S_{\nu}$.

- The eigenvalue set $\tilde{S}_{\nu}=(1,-1,-1)$ is always contained as a diagonal generator for all $\Delta\left(12 k^{2}\right)$ groups and can be chosen as $S_{\nu}=W(2 k, 0, k)$. Hence the texture I with two degenerate and one massless neutrino can follow in any $\Delta\left(12 k^{2}\right)$. The smallest such group is $\Delta(12)=A_{4}$ which is one of the most studied flavour symmetry from other points of view [43-52].

- The set $\tilde{S}_{\nu}=( \pm i, \pm i,-1)$ arises only for $N$ multiple of 4 , i.e. in case of groups $\Delta\left(48 l^{2}\right)$, $l=1,2 \ldots$ These groups also contain a $\tilde{S}_{\nu}$ satisfying the second of eq. (2.3). Thus textures I, II, IV are possible for all $\Delta\left(48 l^{2}\right)$ groups.

- The set $\tilde{S}_{\nu}=\left(\lambda,-\lambda^{*},-1\right)$ with $\lambda \neq \pm 1, \pm i$ and the associated texture III is viable in $\Delta\left(12 k^{2}\right)$ with $k \geq 3$.

Let us now turn to the mixing pattern allowed within the $\Delta\left(12 k^{2}\right)$ groups. $S_{\nu}$ has to be a diagonal operator identified above. Then $T_{l}$ can be any other diagonal operator $W(2 k, p, q)$ or any of $R(2 k, p, q)$ or $V(2 k, p, q)$. In the former case, $U_{l}=\mathbb{1}$, where $\mathbb{1}$ denotes a $3 \times 3$ identity matrix. The neutrino mixing in this case coincides with $V_{\nu}$ diagonalizing any of the four textures of $\tilde{M}_{\nu}$ giving $U_{\mathrm{PMNS}}=V_{\nu}$. None of the allowed $V_{\nu}$ are suitable to give the correct mixing pattern with a non-zero $\theta_{13}$. Thus, $T_{l}$ needs to be any of the non-diagonal element $R, V$. The matrices $V_{R, V}$ diagonalizing $R, V$ are given by

$$
\begin{aligned}
& V_{R}(N, p, q)=\operatorname{diag} .\left(1, \eta^{q}, \eta^{-p}\right) U_{\omega}, \\
& V_{V}(N, p, q)=\operatorname{diag} .\left(1, \eta^{-p}, \eta^{-p-q}\right) U_{\omega}^{*},
\end{aligned}
$$

where,

$$
U_{\omega}=\frac{1}{\sqrt{3}}\left(\begin{array}{ccc}
1 & 1 & 1 \\
1 & \omega^{2} & \omega \\
1 & \omega & \omega^{2}
\end{array}\right)
$$

The final mixing matrix depends upon the choice of specific texture for $\tilde{M}_{\nu}$. Consider the texture I which arises within all the $\Delta\left(12 k^{2}\right)$ groups. $U_{\nu}=V_{\nu}$ in this case is given by eq. (2.7) and $U_{\mathrm{PMNS}}=V_{R, V}^{\dagger} V_{\nu}$. Since a neutrino pair is degenerate, the solar mixing angle $\theta_{12}$ remains undetermined in the symmetry limit. This is reflected by the presence of an unknown angle $\phi$ in eq. (2.7). In this case, the neutrino mass hierarchy is inverted 
and the third column of $U_{\mathrm{PMNS}} \equiv U$ needs to be identified with the massless state. It is independent of the angle $\phi$. We get for $T_{l}=R(N, p, q)$,

$$
U_{i 3}=\frac{1}{\sqrt{3}}\left(c e^{-i \beta} \eta^{p+q}-s, c \omega e^{-i \beta} \eta^{q+p}-s \omega^{2}, c \omega^{2} e^{-i \beta} \eta^{q+p}-s \omega\right)^{T}
$$

with $\eta=e^{\frac{\pi i}{k}}$ for the group $\Delta\left(12 k^{2}\right) . p, q$ take discrete values $0 \ldots 2 k-1$ in above equation while $\beta$ and $\theta$ are unknown quantities appearing in the neutrino mixing matrix eq. (2.7). The entries in $U_{i 3}$ can be permuted by reordering the eigenvalues of $T_{l}$. We will identify the minimum of $\left|U_{i 3}\right|^{2}$ with $s_{13}^{2}$. If the minimum of the remaining two is identified with $c_{13}^{2} s_{23}^{2}$ then one will get a solution with the atmospheric mixing angle $\theta_{23} \leq 45^{\circ}$. In the converse case, one will get a solution $\geq 45^{\circ}$. The experimental values of the leptonic angles are determined through fits to neutrino oscillation data [53-55]. Throughout, we shall specifically use the fits presented in [53] for definiteness. The texture I corresponds to the inverted hierarchy and the best fit values and $3 \sigma$ ranges appropriate for this case are given [53] by:

$$
\begin{aligned}
& \sin ^{2} \theta_{12}=0.308(0.259-0.359), \\
& \sin ^{2} \theta_{23}=0.455(0.380-0.641), \\
& \sin ^{2} \theta_{13}=0.0240(0.0178-0.0298) .
\end{aligned}
$$

Let us mention salient features of results following from eq. (3.6).

- It is always possible to obtain correct $\theta_{13}, \theta_{23}$ by choosing unknown quantities $\theta$ and $\beta$ of $\tilde{M}_{\nu}$. This should be contrasted with situation found in [22] which used neutrino symmetry instead of antisymmetry to obtain a degenerate pair of neutrinos. As discussed there, none of the $\Delta\left(3 N^{2}\right)$ groups could simultaneously account for the values of $\theta_{13}, \theta_{23}$ within $3 \sigma$.

- It is possible to obtain more definite predictions by choosing specific values of $\theta$ and or $\beta$. In contrast to $\theta$ and $\beta$ which are unknown, the choice of $p, q$ is dictated by the choice of $T_{l}$ and it is possible to consider any specific choice of $p, q$ in the range $0, \ldots N-1$. Consider a very specific choice of real $\tilde{M}_{\nu}$, i.e. $\beta=0$ and a residual symmetry $T_{l}=E^{2}$ corresponding to putting $p=q=0$ in eq. (3.6). This equation in this case gives a prediction $\left|U_{23}\right|=\left|U_{33}\right|$ which holds for all values of $\theta$. This relation is equivalent to a maximal $\theta_{23}$ which lies within the $1 \sigma$ range of the global fits [53]. $\theta$ then can be chosen to get the correct $\theta_{13}$. Since the specific choice $p=q=0$ is allowed within all the $\Delta\left(12 k^{2}\right)$ groups, all of them can predict the maximal $\theta_{23}$ and can accommodate correct $\theta_{13}$.

- The relation $\left|U_{23}\right|=\left|U_{33}\right|$ does not hold for a complex $\eta^{p+q}$ even if $\beta=0$. Such choices of $T_{l}$ give departures from maximality in $\theta_{23}$. It is then possible to reproduce both the angles correctly by choosing $\theta$. This is non-trivial since a single unknown $\theta$ determines both $\theta_{13}$ and $\theta_{23}$ for a specific choice of group (i.e. $N$ ) and a residual symmetry $T_{l}$ (i.e. $p$ and $q$ ). The resulting prediction can be worked out numerically 


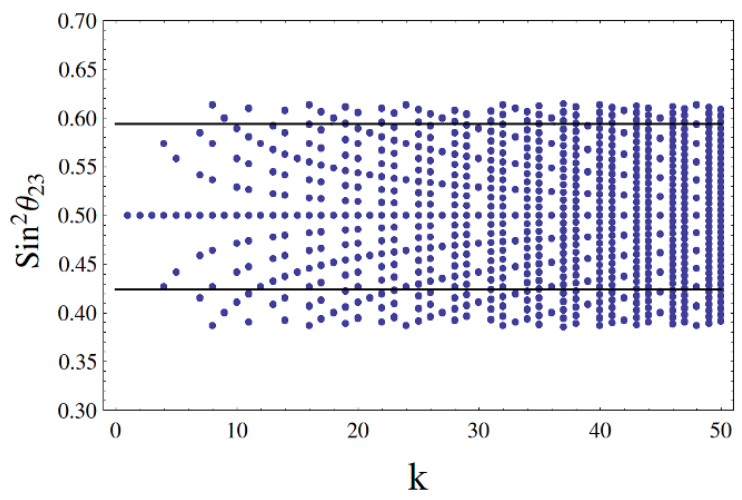

Figure 1. Predictions for $\sin ^{2} \theta_{23}$ for the groups $\Delta\left(12 k^{2}\right)$ as a function of $k$ when $\sin ^{2} \theta_{13}$ is allowed to vary within the $1 \sigma$ range as obtained through global fits in [53]. Horizontal lines show $1 \sigma$ limits on $\sin ^{2} \theta_{23}$.

by varying $p, q, N$ over the allowed integer values and $\theta$ over continuous range from 0 to $2 \pi$. Values of $s_{23}^{2}$ obtained this way are depicted in figure 1 . This is obtained by requiring that $s_{13}^{2}$ lies within the allowed $1 \sigma$ range. The phase $\beta$ is put to zero. It is seen from the figure 1 that all the $\Delta\left(12 k^{2}\right)$ groups always allow maximal $\theta_{23}$ as already discussed. But solutions away from maximal are also possible for $k \geq 4$. The minimal group capable of doing this is $\Delta(192)$. The next group $\Delta(300)$ can lead to near to the best fit values of the parameters. Specifically the choice $T_{l}=R(10,0,7)$, $S_{\nu}=W(10,0,5)$ within the group and $\theta \sim 54.3^{\circ}$ gives $s_{13}^{2} \sim 0.024$ and $s_{23}^{2} \sim 0.442$ to be compared with the best fit values 0.024 and 0.455 in [53].

- $p, q$ can only be zero or 1 and $\eta$ is real for the smallest group $\Delta(12)=A_{4}$. In this case, one immediately gets the prediction $\theta_{23}=\frac{\pi}{4}$ for $\beta=0 . \mu-\tau$ symmetry is often used to predict the maximal $\theta_{23}$. This is not even contained in $A_{4}$ which has only even permutations of four objects. Still the use of antisymmetry rather than symmetry allows one to get the maximal $\theta_{23}$ and it also accommodates a non-zero $\theta_{13}$ within $A_{4}$. This should be contrasted with the situation obtained in case of the use of symmetry condition eq. (1.2) instead of (1.3). It is known that in this case $A_{4}$ group gives democratic value $\frac{1}{3}$ for $s_{13}^{2}$, see for example [6].

We now argue that the other three textures though possible within $\Delta\left(12 k^{2}\right)$ groups do not give the the correct mixing pattern. Texture II has one massless and in general two non-degenerate neutrinos. This texture can give both the normal and the inverted hierarchy. The mixing matrix $V_{\nu}$ is block-diagonal with a $2 \times 2$ matrix giving mixing among two massive states. Given this form for $V_{\nu}$ and a general $U_{l}$ as given in eq. (3.4), one finds that the case with inverted hierarchy leads to the prediction $\sin ^{2} \theta_{13}=\frac{1}{3}$ while the normal hierarchy gives instead $\cos ^{2} \theta_{13} \cos ^{2} \theta_{12}=\frac{1}{3}$. Neither of them come close to their experimental values.

The texture III having degenerate pair corresponds to the inverted hierarchy. $V_{\nu}$ in this case is block diagonal with an unknown solar angle. Given the most general form, 
eq. (3.4) for $V_{l}$ one obtains once again the wrong prediction $\sin ^{2} \theta_{13}=\frac{1}{3}$ ruling out this texture as well. Likewise, texture IV also gets ruled out. This corresponds to a diagonal $\tilde{M}_{\nu}$ with $V_{\nu}=\mathbb{1}$ and $\left|U_{\text {PMNS }}\right|=\left|U_{l}\right|$ has the universal structure $|U|=\frac{1}{3} \mathbb{1}$.

To sum up, all the groups $\Delta\left(12 k^{2}\right)$ contain a neutrino antisymmetry operator $S_{\nu}$ and allow a neutrino mass spectrum with two degenerate and one massless neutrino and can reproduce correctly two of the mixing angles $\theta_{13}, \theta_{23}$. The values for the solar angle and the solar scale have to be generated by small perturbations within these group. We shall study an example based on the minimal group $A_{4}=\Delta(12)$ in this category in section 5 .

\section{$3.2 \Delta\left(6 N^{2}\right)$}

$\Delta\left(6 N^{2}\right)$ groups are isomorphic to $\left(Z_{N} \times Z_{N}\right) \rtimes S_{3}$ with $N=1,2,3 \ldots$. The $S_{3}$ group in the semi-direct product is generated by $E$ in eq. (3.2) and a matrix

$$
G=-\left(\begin{array}{lll}
1 & 0 & 0 \\
0 & 0 & 1 \\
0 & 1 & 0
\end{array}\right)
$$

The matrices $E, F, G$ provide a faithful irreducible representation of $\Delta\left(6 N^{2}\right)$ [30] and generate the entire group with $6 N^{2}$ elements. $3 N^{2}$ elements generated by $E, F$ give the $\Delta\left(3 N^{2}\right)$ subgroup. The additional $3 N^{2}$ elements are generated from the multiple products of $G$ with elements of $\Delta\left(3 N^{2}\right)$. These new elements can be parameterized by:

$$
\begin{aligned}
& S \equiv S(N, m, n)=-\left(\begin{array}{ccc}
\eta^{m} & 0 & 0 \\
0 & 0 & \eta^{n} \\
0 & \eta^{-m-n} & 0
\end{array}\right), \quad T \equiv T(n, m, n)=-\left(\begin{array}{ccc}
0 & 0 & \eta^{n} \\
0 & \eta^{m} & 0 \\
\eta^{-m-n} & 0 & 0
\end{array}\right), \\
& U \equiv U(n, m, n)=-\left(\begin{array}{ccc}
0 & \eta^{n} & 0 \\
\eta^{-m-n} & 0 & 0 \\
0 & 0 & \eta^{m}
\end{array}\right) .
\end{aligned}
$$

Here $0 \leq(m, n)<N-1$. Since $\Delta\left(3 N^{2}\right)$ is a subgroup of $\Delta\left(6 N^{2}\right)$, the neutrino mass and mixing patterns derived in the earlier section can also be obtained here. But the new elements $S, T, U$ allow more possibilities now. In particular, they allow more elements which can be used as neutrino antisymmetry $S_{\nu}$. To see this, note that the eigenvalues of $S, T, U$ are given by $\left(\eta^{-m / 2},-\eta^{-m / 2},-\eta^{m}\right)$. This can have the required form, eq. (2.3) when $m=0$ or $m=N / 2$. The eigenvalues in respective cases are $(1,-1,-1)$ or $(-i, i, 1)$ and one gets the textures I or IV by using any of $S, T, U$ as neutrino antisymmetry with $m=0$ and $m=N / 2$ respectively. Similarly, possible choices of the charged lepton symmetry $T_{l}$ also increases. It can be any of the six types of elements: $W, R, V$ as before or $S, T, U$. Important difference compared to $\Delta\left(3 N^{2}\right)$ is that the texture I can now be obtained for both odd and even values of $N$ by choosing any of the $S, T, U$ with $m=0$ as neutrino antisymmetry. Texture IV still requires $m=N / 2$ and hence even $N$ for its realization. We determine mixing matrix $U$ for each of these textures and discuss them in turn. 


\begin{tabular}{|c|c|c|c|c|c|}
\hline Case & $S_{\nu}$ & $T_{l}$ & $U_{l}$ & $U_{\nu}$ & $U_{\mathrm{PMNS}}$ \\
\hline 1A & $W(2 k, 0, k)$ & $W(N, p, q)$ & $\mathbb{1}$ & $V_{\nu}$ & $V_{\nu}$ \\
\hline 1B & $W(2 k, 0, k)$ & $P(N, p, q)$ & $V_{P}(N, p, q)$ & $V_{\nu}$ & $V_{P}^{\dagger}(N, p, q) V_{\nu}$ \\
\hline 1C & $W(2 k, 0, k)$ & $Q(N, p, q)$ & $V_{Q}(N, p, q)$ & $V_{\nu}$ & $V_{Q}^{\dagger}(N, p, q) V_{\nu}$ \\
\hline 2A & $P(N, 0, n)$ & $W(N, p, q)$ & $\mathbb{1}$ & $V_{P}(N, 0, n) V_{\nu}$ & $V_{P}(N, 0, n) V_{\nu}$ \\
\hline 2B & $P(N, 0, n)$ & $P^{\prime}(N, p, q)$ & $V_{P^{\prime}}(N, p, q)$ & $V_{P}(N, 0, n) V_{\nu}$ & $V_{P^{\prime}}^{\dagger}(N, p, q) V_{P}(N, 0, n) V_{\nu}$ \\
\hline 2C & $P(N, 0, n)$ & $Q(N, p, q)$ & $V_{Q}(N, p, q)$ & $V_{P}(N, 0, n) V_{\nu}$ & $V_{Q}^{\dagger}(N, p, q) V_{P}(N, 0, n) V_{\nu}$ \\
\hline
\end{tabular}

Table 1. All possible choices of the residual symmetries $S_{\nu}$ and $T_{l}$ within $\Delta\left(6 N^{2}\right)$ groups and the corresponding PMNS mixing matrices. $P, P^{\prime}$ collectively denote any of $S, T, U$ defined in the text. $Q$ denotes $R$ and $V$. The mixing matrices $V_{P}, V_{Q}$ and $V_{\nu}$ appearing above are given in eq. (3.10), eq. (3.4) and eq. (2.7) respectively.

\subsubsection{Texture I}

The residual anti symmetries which lead to texture I can be either (1) $S_{\nu}=W(2 k, 0, k)$ or (2) $S_{\nu}=P(N, 0, n)$ where $P=S, T, U$. The residual symmetry $T_{l}$ of $M_{l} M_{l}^{\dagger}$ can be any elements in the group which we divide in three classes: (A) $T_{l}=W(N, p, q)$, (B) $T_{l}=P(N, p, q)$ and (C) $T_{l}=Q(N, p, q)$. Here and in the following, we use symbols $P$ and $Q$ to collectively denote $P=S, T, U$ and $Q=R, V$. We use the basis as specified in eqs. (3.9), (3.3) for $S_{\nu}, T_{l}$. Then the neutrino mixing matrix is given by $U_{\nu}=V_{\nu}$ in case (1) while it is given by $U_{\nu}=V_{P}(N, 0, n) V_{\nu}$ in case (2). This follows by noting that the texture $\tilde{M}_{\nu}$ given in eq. (2.4) holds in a basis with diagonal $S_{\nu}$ but $S_{\nu}$ in the chosen group basis of eq. (3.9) is non-diagonal in case (2). The neutrino mass matrix in this basis is thus given by $M_{\nu}=V_{P}^{*} \tilde{M}_{\nu} V_{P}^{\dagger}$ where $V_{P}$ diagonalizes $P(N, 0, n)$. The matrix $U_{\nu}$ which diagonalizes $M_{\nu}$ is then given by $U_{\nu}=V_{P}(N, 0, n) V_{\nu}$ where $V_{\nu}$ diagonalizes $\tilde{M}_{\nu}$. Explicitly, $V_{P}^{\dagger}(N, p, q) P(N, p, q) V_{P}(N, p, q)=\operatorname{diag} .\left(\eta^{-p / 2},-\eta^{-p / 2},-\eta^{p}\right)$ with

$$
\begin{aligned}
V_{S}(N, p, q) & =\frac{1}{\sqrt{2}}\left(\begin{array}{ccc}
0 & 0 & \sqrt{2} \\
1 & \eta^{q+p / 2} & 0 \\
-\eta^{-q-p / 2} & 1 & 0
\end{array}\right) ; \quad V_{U}(N, p, q)=\frac{1}{\sqrt{2}}\left(\begin{array}{ccc}
1 & \eta^{q+p / 2} & 0 \\
-\eta^{-q-p / 2} & 1 & 0 \\
0 & 0 & \sqrt{2}
\end{array}\right), \\
V_{T}(N, p, q) & =\frac{1}{\sqrt{2}}\left(\begin{array}{ccc}
1 & \eta^{q+p / 2} & 0 \\
0 & 0 & \sqrt{2} \\
-\eta^{-q-p / 2} & 1 & 0
\end{array}\right) .
\end{aligned}
$$

We have chosen the ordering of columns of $V_{P}$ in such a way that the first column always corresponds to the eigenvalue $\eta^{-p / 2}$. With this ordering one gets the texture I given in eq. (2.4) when $P(N, 0, n)$ is used as neutrino antisymmetry.

The matrices $U_{l}$ diagonalizing $T_{l}$ in three cases above are given in the same basis by $U_{l}=\mathbb{1}, V_{P}(N, p, q), V_{Q}(N, p, q)$ in cases $(\mathrm{A}),(\mathrm{B}),(\mathrm{C})$ respectively where $V_{Q}$ are given in eq. (3.4). Thus we have six (four) different choices for $U_{l}\left(U_{\nu}\right)$ giving in all 24 leptonic mixing matrices $U_{\mathrm{PMNS}}$. We list these choices and the corresponding $U_{\mathrm{PMNS}}$ matrices in table 1 .

Not all of 24 mixing matrices listed in table 1 give independent predictions for the third column of $U_{\text {PMNS }}$ which determines $s_{13}$ and $s_{23}$. We discuss the independent ones below. 
The choice (1A) giving $U_{\mathrm{PMNS}}=V_{\nu}$ has one of the entries zero and thus cannot lead to correct $\theta_{13}$ or $\theta_{23}$. The choice (1C) involves only elements belonging to the $\Delta\left(3 N^{2}\right)$ subgroup and its predictions are already discussed in the previous section. The remaining choices give new predictions.

The case (1B) leads to three different $U_{\mathrm{PMNS}}$. One obtained with $T_{l}=S(N, p, q)$ contains a zero entry in the third column and can be used only as a zeroeth order choice. One gets the following result in $(1 \mathrm{~B})$ if $T_{l}=T(N, p, q)$

$$
\left|U_{23}\right|^{2}=\frac{c^{2}}{2}, \quad\left|U_{33}\right|^{2}=\frac{c^{2}}{2}, \quad\left|U_{13}\right|^{2}=s^{2} .
$$

The ordering of the entries $\left|U_{i 3}\right|^{2}$ can be changed by rearranging the eigenvectors of of $T_{l}$ appearing in $U_{l}$. We have chosen here and below an ordering which is consistent with the values of the parameters $s_{13}^{2}, s_{23}^{2}$ when $U$ is equated with the standard form of the mixing matrix. The result in the third case with $T_{l}=U(N, p, q)$ can be obtained from above by the replacement $s \leftrightarrow c$. All the three entries above follow for all the choices of $p, q$ and the phase $\beta$. The case (1B) in this way gives a universal prediction. Two of the $\left|U_{i 3}\right|^{2}$ are equal within this choice and they correspond to $c_{13}^{2} c_{23}^{2}$ and $c_{13}^{2} s_{23}^{2}$. Equality of the two then implies a $\theta$ independent prediction $\theta_{23}=\frac{\pi}{4} . s_{13}^{2}$ in the above case is then given by $s^{2}$ and can match the experimental value with appropriate choice of the unknown $\theta$. Since the choice of $S_{\nu}$ within (1B) is possible only for even $N$ it follows that all the groups $\Delta\left(24 k^{2}\right)$ lead to a prediction of the maximal atmospheric mixing angle and can accommodate the correct $\theta_{13}$.

The choice (2A) also gives the same result for $\left|U_{i 3}\right|^{2}$ as (1B) with an important difference. The neutrino residual symmetry used in this choice is allowed for all $N$ and not necessarily $N=2 k$. Thus one gets a universal prediction of the maximal $\theta_{23}$ for all $p, q, \theta, \beta$ within all $\Delta\left(6 N^{2}\right)$ groups. The smallest group in this category is the permutation group $S_{4}=\Delta(24)$ which contain symmetries appropriate for both the cases (1B) and (2A).

There are two independent structures within nine possible choices contained in case (2B). The example of the first one is provided by the choice $S_{\nu}=S(N, 0, n)$ and $T_{l}=S(N, p, q)$. The elements in the third column of mixing matrix are given in this case by

$$
\begin{aligned}
\left|U_{23}\right|^{2} & =\frac{1}{4} s^{2}\left|\eta^{n}-\eta^{q+p / 2}\right|^{2}, \\
\left|U_{33}\right|^{2} & =\frac{1}{4} s^{2}\left|\eta^{-n}+\eta^{-q-p / 2}\right|^{2}, \\
\left|U_{13}\right|^{2} & =c^{2} .
\end{aligned}
$$

While this choice does not give universal prediction as in the case (1B) discussed above it still leads to a prediction for $\theta_{23}$ which is independent of the unknown angle $\theta$ and phase $\beta$ :

$$
\tan ^{2} \theta_{23} \quad \text { or } \quad \cot ^{2} \theta_{23}=\frac{\left|\eta^{n}-\eta^{q+p / 2}\right|^{2}}{\left|\eta^{-n}+\eta^{-q-p / 2}\right|^{2}}
$$

This follows from eq. (3.12) when $\left|U_{13}\right|^{2}$ is identified with $s_{13}^{2}$. The predicted $\theta_{23}$ now depends only on the group theoretical factors $N, p, q, n$. 


\begin{tabular}{|c|c|c|c|}
\hline Group & $T_{l}$ & $S_{\nu}$ & Predictions \\
\hline$\Delta\left(24 k^{2}\right)$ & $T(2 k, p, q)$ & $W(2 k, 0, k)$ & $\begin{array}{c}\text { Maximal } \theta_{23} \text { for all } \beta, p, q, n \\
\sin ^{2} \theta_{13}=\sin ^{2} \theta\end{array}$ \\
\hline$\Delta\left(6 N^{2}\right)$ & $W(N, p, q)$ & $P(N, 0, n)$ & $\begin{array}{c}\text { Maximal } \theta_{23} \text { for all } \beta, p, q, n \\
\sin ^{2} \theta_{13}=\cos ^{2} \theta\end{array}$ \\
\hline$\Delta\left(6 N^{2}\right)$ & $S(N, p, q)$ & $S(N, 0, n)$ & $\begin{array}{c}\text { Maximal } \theta_{23} \text { for all } \beta, \frac{|n-q-p / 2|}{N}=\frac{(2 l+1)}{4} \\
\sin ^{2} \theta_{13}=\cos ^{2} \theta\end{array}$ \\
\hline$\Delta(294)$ & $S(7,0,2)$ & $S(7,0,0)$ & $s_{23}^{2}=0.39$ or 0.61 for all $\theta, \beta$ \\
$\cos ^{2} \theta=s_{13}^{2}$
\end{tabular}

Table 2. Some illustrative predictions of the mixing angles $\sin ^{2} \theta_{13}$ and $\sin ^{2} \theta_{23}$ using $\Delta\left(6 N^{2}\right)$ groups as flavour symmetry. $\sin ^{2} \theta_{12}$ remains undetermined due to degeneracy in two of the masses in all these cases.

Unlike (1B), both the maximal and non-maximal values are allowed for $\theta_{23}$ in this case. The former occurs whenever $\cos \frac{2 \pi(n-q-p / 2)}{N}=0$. The latter occurs for other choices. It is possible to find values of parameters which lead to a non-maximal $\theta_{23}$ within the experimental limits. The minimal such choice occurs for $N=7$, i.e. the group $\Delta(294)$ which leads as shown in table 2 to a $\sin ^{2} \theta_{23}$ within the $2 \sigma$ range as given in [53]. The next example of the group $\Delta(486)$ fairs slightly better.

The other prediction of the case $(2 \mathrm{~B})$ is obtained with $S_{\nu}=S(N, 0, n)$ and $T_{l}=$ $T(N, p, q)$. One obtains in this case

$$
\begin{aligned}
\left|U_{23}\right|^{2} & =\frac{1}{4}\left|\sqrt{2} c e^{-i \beta}+s \eta^{q+p / 2}\right|^{2}, \\
\left|U_{33}\right|^{2} & =\frac{1}{4}\left|\sqrt{2} c e^{-i \beta}-s \eta^{-q-p / 2}\right|^{2}, \\
\left|U_{13}\right|^{2} & =\frac{1}{2} s^{2} .
\end{aligned}
$$

In this case, $\theta_{23}$ is necessary non-maximal if $\theta_{13}$ is to be small but non-zero. We may identify, $\left|U_{13}\right|^{2}$ with $s_{13}^{2}$ and fix $s^{2}=2 s_{13}^{2}$. This determines the other two entries of $\left|U_{i 3}\right|^{2}$ for a given $p, q, \beta$. For $p=q=\beta=0$ one obtains $\sin ^{2} \theta_{23}$ either 0.345 or 0.655 . Thus all the $\Delta\left(6 N^{2}\right)$ groups with this specific choice give results close to the $3 \sigma$ range in the global fits. This prediction can be improved by turning on $\beta$ or choosing different $T_{l}$. An example 
based on the group $\Delta(150)$ giving $\sin ^{2} \theta_{23}$ close to the best fit value [53] is shown in the table 2 .

Predictions of the case (2C) can also be similarly worked out. Six different $U_{\text {PMNS }}$ are associated with this choice but not all give different predictions for the third column. One of the independent structures corresponds to choosing $T_{l}=R(N, p, q)$ and $S_{\nu}=S(N, 0, n)$. The $U_{\mathrm{PMNS}}=V_{R}^{\dagger} V_{S} V_{\nu}$ gives

$$
\begin{aligned}
& \left|U_{13}\right|^{2}=\frac{1}{6}\left|-s\left(\eta^{p}+\eta^{n-q}\right)+\sqrt{2} c e^{-i \beta}\right|^{2}, \\
& \left|U_{23}\right|^{2}=\frac{1}{6}\left|-s\left(\eta^{p} \omega+\eta^{n-q} \omega^{2}\right)+\sqrt{2} c e^{-i \beta}\right|^{2}, \\
& \left|U_{33}\right|^{2}=\frac{1}{6}\left|-s\left(\eta^{p} \omega^{2}+\eta^{n-q} \omega\right)+\sqrt{2} c e^{-i \beta}\right|^{2} .
\end{aligned}
$$

Rest of the choices within $(2 \mathrm{C})$ differ from the above only in the powers of $\eta$. Their predictions can be obtained from the above by choosing different values of $p, q, n$.

Eq. (3.14) gives $s_{13}, s_{23}$ withing $3 \sigma$ range for a suitable choice of $N, p, q, \theta, \beta$. In particular, one predicts a maximal $\theta_{23}$ if $p=q=\beta=0$ as in the earlier cases. But now the maximal value of $\theta$ also becomes a viable choice for all the groups $\Delta\left(6 N^{2}\right)$. This makes the choice in this class particularly interesting since such value of $\theta$ can be forced by some additional symmetry. With the choice $T_{l}=E^{2}$ corresponding to $p=q=0$, eq. (3.14) gives for $n=\beta=0$,

$$
\theta_{23}=\frac{\pi}{4}, \quad s_{13}^{2}=\frac{1}{3}|c-\sqrt{2} s|^{2} .
$$

The maximal value of $\theta$ then leads to $s_{13}^{2} \sim 0.029$ which is close to $2 \sigma$ range as obtained in [53]. One can obtain a better solution with a different choice for $p$ and $q$ and $\theta$. One particular solution based on the group $\Delta(150)$ is shown in the table 2 .

\subsubsection{Texture IV}

The diagonal texture IV given in eq. (2.8) can be realized in $\Delta\left(6 N^{2}\right)$ for even $N$ with the choice $S_{\nu}=P(2 k, k, n)$. This texture has two non-degenerate and one massless neutrino. Thus both the normal and the inverted hierarchies are possible. The massless state has to be identified with the third (first) column of the mixing matrix for the inverted (normal) hierarchy. The neutrino mixing matrix $U_{\nu}$ in this case is given by the matrix which diagonalizes $P(2 k, k, n)$. This is given for the inverted hierarchy by $V_{P}(2 k, k, n)$ as defined in eq. (3.10). For the normal hierarchy, one instead gets $U_{\nu}=V_{P}(2 k, k, n) Z_{13}$ where $Z_{13}$ exchanges the first and the third column of of the mixing matrix obtained in case of the inverted hierarchy. Possible choice of $T_{l}$ can be any of the six types of generators and corresponding mixing matrices $U_{l}$ are the same as given in table 1 with the choice $(2 \mathrm{~A}),(2 \mathrm{~B}),(2 \mathrm{C})$. It is then straightforward to work out the final mixing matrices $U_{\text {PMNS. As the massless }}$ state in the basis with diagonal $S_{\nu}$ is given by $(1,0,0)^{T}$ and its cyclic permutation for $S_{\nu}=S, T, U$, the third column of the $U_{\mathrm{PMNS}}$ is given by $\left(U_{\mathrm{PMNS}}\right)_{i 3}=\left(U_{l}^{\dagger}\right)_{i 1},\left(U_{l}^{\dagger}\right)_{i 2},\left(U_{l}^{\dagger}\right)_{i 3}$ when $S_{\nu}=S, T, U$. It follows from the structure of $U_{l}$ that the third column has either one or two zero entries or all elements have equal magnitudes. The same applies to the first column of $U_{\mathrm{PMNS}}$ in case of the normal hierarchy. In either case, the texture IV cannot give phenomenologically consistent result at the zeroeth order. 


\section{More predictive scenario: $Z_{2} \times Z_{2}$ symmetry}

The neutrino mass matrix $M_{\nu}$ has been assumed so far to be antisymmetric with respect to only one $S_{\nu}$. This fails in determining $\tilde{M}_{\nu}$ completely in case of the texture I which still has two unknown quantities $\theta$ and $\beta$. The situation changes if $M_{\nu}$ is assumed to be symmetric or antisymmetric with respect to one more generator. We give here an example in which an additional residual symmetry of $\tilde{M}_{\nu}$ determines it completely apart from an overall complex mass scale. We use a generator $S_{\nu}^{\prime}$ commuting with $S_{\nu}$ for this purpose. It should be such that $S_{\nu}, S_{\nu}^{\prime}$ and $T_{l}$ together are contained in some $G_{f} . M_{\nu}$ may be antisymmetric with respect to transformation by $S_{\nu}^{\prime}$ also. In this case, it will be symmetric with respect to the product $S_{\nu} S_{\nu}^{\prime}$. Instead we assume that $S_{\nu}^{\prime}$ is a symmetry of $M_{\nu}$, i.e.

$$
S_{\nu}^{\prime T} M_{\nu} S_{\nu}^{\prime}=M_{\nu}
$$

We can transform above equation to the basis with a diagonal $S_{\nu}$ by defining ${\tilde{S^{\prime}}}_{\nu} \equiv$ $V_{S_{\nu}}^{\dagger} S_{\nu}^{\prime} V_{S_{\nu}}$. In this basis, we get

$$
\tilde{S}_{\nu}{ }^{T} \tilde{M}_{\nu}{\tilde{S_{\nu}}}^{\prime}=\tilde{M}_{\nu}
$$

As before, we demand $\tilde{S_{\nu}^{\prime}}$ to be contained in $\mathrm{SU}(3)$. If it is diagonal, then $\tilde{S}_{\nu}^{\prime}=\operatorname{diag} .\left(\lambda_{1}, \lambda_{2}\right.$, $\lambda_{1}^{*} \lambda_{2}^{*}$ ) with $\lambda_{1,2}$ being roots of unity. Then eq. (4.2) when applied to $\tilde{M}_{\nu}$ in eq. (2.7) implies that either ${\tilde{S_{\nu}}}^{\prime}$ is proportional to identity or $s=0$ or $c=0$. A non-trivial prediction can be obtained if ${\tilde{S_{\nu}}}^{\prime}$ is non-diagonal. Since $\tilde{S}_{\nu}=\operatorname{diag} .(1,-1-1)$, a general $\tilde{S}_{\nu}{ }^{\prime}$ commuting with $\tilde{S}_{\nu}$ should have a block diagonal structure with the lower $2 \times 2$ block non-trivial. This block gets further restricted from the requirement that $S_{\nu}, S_{\nu}^{\prime}, T_{l}$ are elements of some discrete group $G_{f}$. These requirements can be met within the already considered groups $\Delta\left(6 N^{2}\right)$.

Consider the group $\Delta\left(12 k^{2}\right)$. The choice $S_{\nu}=\tilde{S_{\nu}}=W(2 k, 0, k)=\operatorname{diag} .(1,-1,-1)$ within it leads to texture I as already discussed. This commutes with all the discrete symmetries having a general form $S(M, m, n)$ as in eq. (3.9). Thus a viable choice for $S_{\nu}^{\prime}$ is provided by $S_{\nu}^{\prime}=S(M, m, n)$. Note that since $S_{\nu}$ is already diagonal, ${\tilde{S_{\nu}}}^{\prime}=S_{\nu}^{\prime}=$ $S(M, m, n)$. Then eq. (4.2) and the form of $\tilde{M}_{\nu}$ implies a restriction:

$$
m=0, \quad \theta= \pm \frac{\pi}{4}, \quad \beta=\frac{2 \pi n}{M}
$$

which fixes the unknown angle $\theta$ and phase $\beta$. Mixing pattern can be determined by choosing appropriate $T_{l}$ and let us choose $T_{l}=R(N, p, q)$. Since both $T_{l}$ and $S_{\nu}$ are contained in $\Delta\left(12 k^{2}\right)$ mixing pattern is determined by the corresponding eq. (3.6) but now with $\theta$ and $\beta$ satisfying eq. (4.3) which follows from the inclusion of $S_{\nu}^{\prime}$ as a residual symmetry. We can vary $p, q, M, N, n$ in eq. (3.6) and look for a viable choice. Consider $M=N$ in which case $T_{l}, S_{\nu}, S_{\nu}^{\prime}$ are contained in $\Delta\left(6 N^{2}\right)$. By varying $p, q, N$ one finds that the minimum group giving acceptable $\theta_{13}, \theta_{23}$ is $\Delta(600)$ corresponding to $N=10$. One possible set of residual symmetries within $\Delta(600)$ is given by

$$
S_{\nu}=W(10,0,5), \quad T_{l}=R(10,4,0), \quad S_{\nu}^{\prime}=S(10,0,0) .
$$


With this choice, the $S_{\nu}^{\prime}$ coincides with the $\mu$ - $\tau$ symmetry and eqs. (3.6), (4.3) give a prediction

$$
s_{13}^{2} \approx 0.029, \quad s_{23}^{2} \approx 0.38 \text { or } 0.62
$$

to be compared with the $3 \sigma$ region given in eq. (3.7).

\section{An $A_{4}$ model with flavour antisymmetry}

Our discussion so far has been at the group theoretical level. We now present an explicit realization of flavour antisymmetric neutrino mass matrix using $A_{4}$ as an example. $A_{4}$ has been extensively used for several different purposes, for obtaining degenerate neutrinos [43, 44], to realize tri-bimaximal mixing $[45,48]$ for obtaining maximal CP phase $\delta[46,47,49$, $51,52]$ or to obtain texture zeros [50] in the leptonic mass matrices. As we discuss here, it also provides a viable alternative to get a massless and two quasi degenerate neutrinos with correct mixing pattern. In the following, we discuss the required symmetry, Higgs content and obtain the vacuum needed to obtain antisymmetry. We also discuss possible perturbations which can split the degenerate pair and lead to the solar scale and mixing angle.

The group theory of $A_{4}$ is discussed extensively in many papers. We follow the basis choice as given for example in [48]. In this basis, all the 12 elements of $A_{4}=\Delta(12)$ can be generated from the two elements $E$ and $F$ defined in eq. (3.2) with $\eta=-1$. We will use the following product rule between two three dimensional representations $x=\left(x_{1}, x_{2}, x_{3}\right)$ and $y=\left(y_{1}, y_{2}, y_{3}\right)$ :

$$
x \times y=1+1^{\prime}+1^{\prime \prime}+\left(\begin{array}{c}
x_{2} y_{3}+x_{3} y_{2} \\
x_{3} y_{1}+x_{1} y_{3} \\
x_{1} y_{2}+x_{2} y_{1}
\end{array}\right)_{3 S}+\left(\begin{array}{c}
x_{2} y_{3}-x_{3} y_{2} \\
x_{3} y_{1}-x_{1} y_{3} \\
x_{1} y_{2}-x_{2} y_{1}
\end{array}\right)_{3 A}
$$

with $1=x_{1} y_{1}+x_{2} y_{2}+x_{3} y_{3}, 1^{\prime}=x_{1} y_{1}+\omega x_{2} y_{2}+\omega^{2} x_{3} y_{3}, 1^{\prime \prime}=x_{1} y_{1}+\omega^{2} x_{2} y_{2}+\omega x_{3} y_{3}$.

The explicit model presented below is based on the flavour symmetry $A_{4} \times Z_{3} \times Z_{5}$. The added symmetry $Z_{3} \times Z_{5}$ plays an important role in restricting the structure of the model in a way that leads to correct vacuum alignment and the required antisymmetric $M_{\nu}$. The $A_{4}$ symmetry determines the mixing angle structure obtained group theoretically in the earlier section.

The model uses the following ingredients (1) supersymmetry with unbroken $R$ symmetry (2) flavon fields with zero $R$-charge which break the $A_{4}$ symmetry and (3) the driving fields with $R=2$ which appear linearly in suprepotential and lead to correct vacuum alignment among flavons. $R$ symmetry is assumed to be unbroken and driving fields have zero vacuum expectation values. These ingredients have been used in several papers to solve the difficult vacuum alignment problem in case of flavour symmetry, see for a review and references [18]. We use the same mechanism to get antisymmetry. The quantum numbers of the required flavonic superfields under $A_{4} \times Z_{3} \times Z_{5}$ symmetry are listed in table 3 and that of the driving fields in table 4. The MSSM Higgs fields $H_{u}, H_{d}$ and triplet $\Delta$ are invariant under $A_{4} \times Z_{3} \times Z_{5}$ symmetry. The model also needs and additional triplet superfield $\bar{\Delta}$ for consistency. 


\begin{tabular}{|c|c|c|c|c|c|c|c|}
\hline Fields & $l_{L}$ & $\left(e^{c}, \mu^{c}, \tau^{c}\right)$ & $\chi_{e}$ & $\chi_{1}$ & $\chi_{2}$ & $\xi_{1}$ & $\xi_{2}$ \\
\hline$A_{4}$ & 3 & $\left(1,1^{\prime}, 1^{\prime \prime}\right)$ & 3 & 3 & 3 & 1 & 1 \\
\hline$Z_{3}$ & $\omega$ & $\omega^{2}$ & 1 & $\omega$ & $\omega$ & 1 & 1 \\
\hline$Z_{5}$ & 1 & $\beta^{3}$ & $\beta^{2}$ & $\beta$ & $\beta^{4}$ & $\beta^{4}$ & $\beta$ \\
\hline
\end{tabular}

Table 3. Transformation properties of leptons and the required flavon fields under the symmetry group $A_{4} \times Z_{3} \times Z_{5} . \omega$ and $\beta$ satisfy $\omega^{3}=\beta^{5}=1$.

\begin{tabular}{|c|c|c|c|c|c|c|c|}
\hline Driving Fields & $\left(\sigma_{e}^{0}, \sigma_{\mu}^{0}, \sigma_{\tau}^{0}\right)$ & $\chi_{1}^{0}$ & $\chi_{2}^{0}$ & $\sigma_{\nu}^{0}$ & $\sigma_{\xi}^{0}$ & $\sigma_{1}^{\prime 0}$ & $\sigma_{2}^{\prime 0}$ \\
\hline$A_{4}$ & $\left(1,1^{\prime \prime}, 1^{\prime}\right)$ & 3 & 3 & 1 & 1 & 1 & 1 \\
\hline$Z_{3}$ & 1 & $\omega$ & $\omega$ & $\omega$ & 1 & $\omega$ & $\omega$ \\
\hline$Z_{5}$ & $\beta$ & $\beta^{3}$ & $\beta^{2}$ & 1 & 1 & $\beta^{3}$ & $\beta^{2}$ \\
\hline
\end{tabular}

Table 4. Transformation properties of the required driving fields under the symmetry group $A_{4} \times Z_{3} \times Z_{5} . \omega$ and $\beta$ satisfy $\omega^{3}=\beta^{5}=1$.

The complete superpotential consists of several parts. We discuss significance of each of them below.

$$
\begin{aligned}
W_{l} & =\frac{H_{d}}{M}\left(h_{e}\left(l_{L} \chi_{e}\right)_{1} e_{R}+h_{\mu}\left(l_{L} \chi_{e}\right)_{1^{\prime \prime}} \mu_{R}+h_{\tau}\left(l_{L} \chi_{e}\right)_{1^{\prime}} \tau_{R}\right), \\
W_{\nu} & =\frac{1}{2 M^{2}}\left(l_{L}^{T} C \Delta l_{L}\right)_{3_{S}}\left(h_{1} \chi_{1} \xi_{1}+h_{2} \chi_{2} \xi_{2}\right), \\
W_{l d} & =\beta_{\mu}\left(\chi_{e} \chi_{e}\right)_{1^{\prime}} \sigma_{\mu}^{0}+\beta_{\tau}\left(\chi_{e} \chi_{e}\right)_{1^{\prime \prime}} \sigma_{\tau}^{0}, \\
W_{\nu d} & =\delta_{1}\left(\chi_{1} \chi_{1} \chi_{1}^{0}\right)_{1}+\delta_{2}\left(\chi_{2} \chi_{2} \chi_{2}^{0}\right)_{1}+\delta_{3}\left(\chi_{1} \chi_{2}\right)_{1} \sigma_{\nu}^{0}+\delta_{4}\left(\xi_{1} \xi_{2}-\mu_{\xi}^{2}\right) \sigma_{\xi}^{0}, \\
W_{d}^{\prime} & =\beta_{e}\left(\left(\chi_{e} \chi_{e}\right)_{1}-\mu_{e} \xi_{1}\right) \sigma_{e}^{0}+\beta_{1}^{\prime}\left(\left(\chi_{1} \chi_{1}\right)_{1}-\mu_{1}^{2}\right) \sigma_{1}^{\prime 0}+\beta_{2}^{\prime}\left(\left(\chi_{2} \chi_{2}\right)_{1}-\mu_{2}^{2}\right) \sigma_{2}^{\prime 0} .
\end{aligned}
$$

The subscript $a$ in $(\ldots)_{a}$ in the above equations labels the $A_{4}$ representation according to which the quantity (...) transforms. $C$ is the charge conjugation matrix. The cut-off scale $M$ and the flavon vacuum expectation values generate the effective Yukawa couplings in the model. We have kept only the leading order terms in the above superpotential.

$W_{l}$ and $W_{\nu}$ respectively determine $M_{l}$ and $M_{\nu}$. Note that the assignments given in table 3 forbid terms having only one flavon field in $W_{\nu}$. Thus neutrino masses contain an extra flavon and suppression factor $M$ compared to the charged lepton masses. The residual symmetry properties of the leptonic mass matrices are determined from the above superpotential by the flavon vacuum expectation values (vev) at the minimum. We shall show that these vev lead to the required symmetries in accordance with the discussion given in section 2. The symmetry $T_{l}$ of $M_{l} M_{l}^{\dagger}$ is obtained as $E$ or $E^{2}$. Only possible choice within $A_{4}$ for $S_{\nu}$ leading to flavour antisymmetry is given by $F$ or its cyclic permutations and this can come from the minimization of $W_{\nu d}$.

The minimum of the potential is obtained in the supersymmetric limit by setting $F$ terms corresponding to each superfield to zero. $W_{l d}$ and $W_{\nu d}$ are responsible for the vacuum alignment in the model. Consider derivatives of $W_{\nu d}$ with respect to each component $i=1,2,3$ of triplets $\chi_{1,2}^{0}$ and $\sigma_{\nu}^{0}$

$$
F_{\chi_{1 i}^{0}}=F_{\chi_{2 i}^{0}}=F_{\sigma_{\nu}^{0}}=0
$$


Using the product rules as given in eq. (5.1), one finds that the first two conditions imply that at most one component of the each of the flavon fields $\chi_{1,2}$ can have non-zero vev. $^{2}$ The additional constraint $F_{\sigma_{\nu}^{0}}$ then implies that $\left\langle\chi_{1}\right\rangle$ and $\left\langle\chi_{2}\right\rangle$ form an orthogonal pair of vectors. Thus only possible non-zero vev for these fields are given by

$$
\left\langle\chi_{1}\right\rangle=v\left(\begin{array}{l}
0 \\
0 \\
1
\end{array}\right), \quad\left\langle\chi_{2}\right\rangle=u\left(\begin{array}{l}
0 \\
1 \\
0
\end{array}\right)
$$

or

$$
\left\langle\chi_{1}\right\rangle=v\left(\begin{array}{l}
0 \\
0 \\
1
\end{array}\right), \quad\left\langle\chi_{2}\right\rangle=u\left(\begin{array}{l}
1 \\
0 \\
0
\end{array}\right)
$$

and their cyclic permutations which are related to the above by $A_{4}$ symmetry. The last term of $W_{\nu d}$ assures through $F_{\sigma_{\xi}^{0}}=0$ that the fields $\xi_{1,2}$ assume non-zero vev. Flavon vev given in eq. (5.8) displays antisymmetry

$$
F\left\langle\chi_{1,2}\right\rangle=-\left\langle\chi_{1,2}\right\rangle .
$$

These vevs when substituted in eq. (5.3) lead to a neutrino mass matrix given by texture I, eq. (2.4) with $\tan \theta=\frac{\left|h_{2}\left\langle\xi_{2}\right\rangle u\right|}{\left|h_{1}\left\langle\xi_{1}\right\rangle v\right|}, \beta=\operatorname{Arg}\left(h_{2}\left\langle\xi_{2}\right\rangle u h_{1}^{*}\left\langle\xi_{1}^{*}\right\rangle v^{*}\right)$ and appropriately defined $m_{0}$. Similarly, the vev given in eq. (5.9) display similar antisymmetry with respect to $S_{\nu}=$ diag. $(-1,1,-1)$ which is also an element of $A_{4}$ and leads to a permutation of the texture I. The supersymmetric solution corresponding to $F$-terms $F_{\sigma_{\mu}^{0}}=F_{\sigma_{\tau}^{0}}=0$ lead from eq. (5.4)

$$
\left(\chi_{e} \chi_{e}\right)_{1^{\prime}}=\left(\chi_{e} \chi_{e}\right)_{1^{\prime \prime}}=0 \text {. }
$$

A solution of these two equations is given by

$$
\left\langle\chi_{e 1}\right\rangle^{2}=\left\langle\chi_{e 2}\right\rangle^{2}=\left\langle\chi_{e 3}\right\rangle^{2} .
$$

Equality of vev of all three components is one of the solutions of the above equation. This leads to an $M_{l} M_{l}^{\dagger}$ having a residual symmetry $T_{l}=E, E^{2}$ as has been discussed in several papers on $A_{4}$. Other solutions of eq. (5.10) corresponds to equal magnitudes but relative minus sign between one or two components. Such solutions are invariant under $A_{4}$ elements $f_{i} E f_{i}$, with $f_{1}=F, f_{3}=E F E^{2}, f_{2}=E^{2} F E$. The texture I obtained here is diagonalized by $U_{\nu}=V_{\nu}$. The resulting mixing pattern is a special case of eq. (3.6) obtained for $\Delta\left(12 k^{2}\right)$ with $T_{l}=R(2 k, p, q)$ and $S_{\nu}=W(2 k, 0, k)$. The choices $T_{l}=E, f_{i} E f_{i}$ are obtained from above by a suitable allowed value of $p, q$. Consider the case with $T_{l}=E^{2}$ corresponding to $R(2,0,0)$ and $S_{\nu}=F=W(2,0,1)$. Thus $U_{i 3}$ can be obtained by putting $p=q=0$ and $\eta=-1$ in eq. (3.6). As already discussed, this leads to a prediction $\theta_{23}=\frac{\pi}{4}$ for $\beta=0$ independent of the choice of $\theta$. The latter can be chosen to give the correct $\theta_{13}$ while the solar angle and scale remain unpredicted at this stage due to degeneracy in mass.

\footnotetext{
${ }^{2}$ All components of $\chi_{1,2}=0$ is also a solution. Such solutions do not contribute to the leptonic masses and can be avoided in the presence of suitable supersymmetry breaking mass terms. We shall assume throughout that vev of none of the flavon fields are identically zero.
} 
The flavon vev $v, u$ remain undetermined at this stage. The last piece $W_{d}^{\prime}$ of the superpotential given in eq. (5.6) is introduced to fix these vev. The price for this to be paid in the model is introduction of soft $Z_{3} \times Z_{5}$ breaking mass parameters $\mu_{1}$ and $\mu_{2}$. Apart from introduction of these mass parameters, the superpotential given by eqs. (5.2)-(5.6) is the most general superpotntial invariant under the $A_{4} \times Z_{3} \times Z_{5}$ symmetry. The soft breaking masses may result from the neglected higher order terms, e.g. $\mu_{1}^{2}$ may come from $\frac{\xi_{1}}{M} \chi_{2}^{2} \sigma_{1}^{\prime 0}$ and $\mu_{2}^{2}$ may come from $\frac{\xi_{2}}{M} \chi_{1}^{2} \sigma_{2}^{\prime 0}$ allowed in the superpotential by the $A_{4} \times Z_{3} \times Z_{5}$ symmetry. Rather than considering full higher order corrections, we discuss model only at the leading order and regard for the present the masses $\mu_{1,2}^{2}$ as effective soft $Z_{3} \times Z_{5}$ breaking parameters. This is a technically natural assumption.

Perturbations are needed to split the degenerate pair and stabilize the solar angle. We now discuss possible perturbations within the model. One source of perturbation arises from the shift in the vev of $\chi_{1,2}$ which may arise from higher order effects. Consider for example,

$$
\left\langle\chi_{1}\right\rangle=v\left(\begin{array}{c}
\delta v_{1} \\
\delta v_{2} \\
1
\end{array}\right)
$$

where, $\left|\delta v_{1,2}\right| \ll 1$. The $\delta v_{1}$ generates a non-zero 23 elements in $M_{\nu}$, eq. (2.4). The $\delta v_{2}$ corrects the already existing entries $s, c$ and can be absorbed in their redefinition. Zero entries in the diagonal part of $M_{\nu}$ can be generated through an $A_{4}$ singlet contribution $\left(l_{L}^{T} C \Delta l_{L}\right)_{1}$ which can arise for example from from the higher order $A_{4} \times Z_{3} \times Z_{5}$ invariant terms, e.g., $\frac{1}{M^{3}}\left(l^{T} C \Delta l\right)_{1}\left(\chi_{e} \chi_{2}\right)_{1} \xi_{1}$. Motivated by this, we consider the following perturbed neutrino mass matrix.

$$
\tilde{M}_{\nu}=m_{0}\left(\begin{array}{ccc}
\epsilon_{1} & c & s e^{i \beta} \\
c & \epsilon_{1} & \epsilon_{2} \\
s e^{i \beta} & \epsilon_{2} & \epsilon_{1}
\end{array}\right) .
$$

Inclusion all possible non-leading effects may result in more complex $M_{\nu}$ and would also correct $M_{l}$. All these corrections will add more parameters to the model and we assume their contribution to be small. Here we show that two parameters $\epsilon_{1,2}$ are sufficient to reproduce the neutrino mixing and scales correctly. They split the degeneracy and generate the solar scale and angle correctly. For example,

$$
\left(\theta, \beta, \epsilon_{1}, \epsilon_{2}\right)=(0.5904,-0.1818-0.0579,0.1186)
$$

give the following values of the observables

$$
\sin ^{2} \theta_{13} \sim 0.024, \quad \sin ^{2} \theta_{23} \sim 0.455, \quad \sin ^{2} \theta_{12} \sim 0.307, \quad \frac{\Delta_{\odot}}{\left|\Delta_{\text {atm }}\right|} \sim 0.0317
$$

which corresponds to (nearly) best fit values obtained for example with a global fits in [53].

\section{Summary}

The bottom up approach of finding discrete symmetry groups starting with possible symmetries of the residual mass matrices has been successfully used in last several years to 
predict leptonic mixing angles. The residual symmetry assumed in these works leaves the neutrino mass matrix $M_{\nu}$ invariant. We have proposed here a different possibility in which $M_{\nu}$ displays antisymmetry as defined in eq. (1.3) under a residual symmetry. The use of antisymmetry is found to be more predictive than symmetry. It is able to restrict both neutrino masses and mixing angles unlike all the previous works in this category which [115] could predict only mixing angles. Moreover, the antisymmetry condition by itself is sufficient for determining all possible discrete residual antisymmetry operators $S_{\nu}$ residing in $\mathrm{SU}(3)$. This in turn leads to very specific textures of the neutrino mass matrix satisfying antisymmetry condition. These are given by eqs. (2.4), (2.5), (2.6), (2.8).

Just like symmetry, the antisymmetry of $M_{\nu}$ can also come from the spontaneous breaking of some discrete group $G_{f}$. We have demonstrated it through a supersymmetric model based on the group $A_{4} \times Z_{3} \times Z_{5}$. Just like its counterparts in the case of symmetry $[18,56-58]$, the present model leading to antisymmetry also needs an elaborate set of flavons and driving fields.

We studied the mixing angle predictions in the specific context of the groups $\Delta\left(3 N^{2}\right)$ and $\Delta\left(6 N^{2}\right)$. The main results obtained are:

- Only the groups $\Delta\left(12 k^{2}\right)$ with $k=1,2 \ldots$ and all $\Delta\left(6 N^{2}\right)$ groups contain the residual antisymmetry operator.

- Of the four possible neutrino mass textures allowed by antisymmetry, only texture I having one massless and two degenerate neutrinos can lead to correct mixing pattern. This case provides a very good zeroeth order approximation to reality if the neutrino mass hierarchy is inverted.

- There always exists within these groups residual symmetries of $M_{l} M_{l}^{\dagger}$ and $M_{\nu}$ such that the atmospheric neutrino mixing angle is maximal. Correct value of $\theta_{13}$ can be accommodated by choosing the unknown angle in eq. (2.4) appropriately. There also exists other choices of residual symmetries which for some groups allow non-maximal values of the atmospheric neutrino mixing angle as well. The results of various cases are summarized in figure 1 and table 2.

- The successful texture I still has two free parameters apart from an overall mass scale. But as we have shown here, predicted atmospheric mixing angle in many cases is independent of these unknowns. The reactor angle $\theta_{13}$ depends on it but it is possible to determine these unknown also by enlarging the residual symmetry and we have given an example of a $Z_{2} \times Z_{2}$ residual symmetry which can determine the complete neutrino mass matrix up to an overall scale in terms of group theoretical parameters alone and have identified $\Delta(600)$ as a possible group which can give correct $\theta_{13}$ and $\theta_{23}$ with this symmetry.

We end this section with a comparison of the present work with some earlier relevant works.

- The texture I, eq. (2.4) has been extensively studied since long in the context of $L_{e}-L_{\mu}-L_{\tau}$ global symmetry which implies it, see for example [59] and references 
therein. Imposition of this symmetry on the charged lepton mass matrix $M_{l}$ makes it diagonal after redefinition of $\theta$ appearing in (2.4). Thus the matrix $V_{\nu}$ as given in eq. (2.7) corresponds to the final mixing matrix which is now not allowed by the present experimental constraints. This is not the case here since the $M_{l} M_{l}^{\dagger}$ is non-trivial with the imposed discrete symmetry.

- Neutrino mass matrix displaying a specific flavour antisymmetry namely, $\mu-\tau$ antisymmetry was studied in [60]. This antisymmetry was assumed there to hold in the neutrino flavour basis. In our terminology, this would correspond to study of a specific example within the choice $(2 \mathrm{~A})$ discussed in section 3.2.1. The structure of the neutrino mass matrix and the mixing angle predictions obtained here for this choice agrees with ref. [60] after suitable basis change. The study presented here is not limited to the $\mu-\tau$ antisymmetry but encompasses all possible antisymmetry operators within $\mathrm{SU}(3)$ and leads to many new phenomenological predictions.

- The antisymmetry condition, eq. (1.3), can be converted to the usually assumed symmetry condition by redefining the operator $S_{\nu} \rightarrow i S_{\nu}$. The new operator does not however have unit determinant and would belong to a U(3) group. The occurrence of massless state within such group with condition, eq. (1.2) was discussed in [23, 24]. The residual symmetry operators used there had eigenvalues $(\eta, 1,-1)$ (or its permutations) with $\eta \neq \pm 1$. This coincides with eigenvalues of $i S_{\nu}$ for texture IV when $\eta=i$. Only texture IV was considered in $[23,24]$ and it was shown there that a large class of DSG of U(3) imply $\sin ^{2} \theta_{13}$ to be either 0 or $\frac{1}{3}$ with condition (2). The same conclusion is found to be true here with eq. (1.3) and texture IV in case of the group series $\Delta\left(3 N^{2}\right)$ and $\Delta\left(6 N^{2}\right)$.

- It is possible to obtain a degenerate pair of neutrinos using symmetry condition, eq. (1.2) and DSG of SU(3). This was studied for the finite von-Dyck groups in [21] and for all DSG of SU(3) having three dimensional IR in [22]. Here, the third state is not implied to be massless. The case of one massless and two degenerate neutrinos can follow from the symmetry condition if DSG of U(3) are used. This was also discussed in [22]. The successful examples found in these two works are different from here because of the difference in the assumed residual symmetries. The cases studied in the context of DSG of $\mathrm{SU}(3)$ and $\mathrm{U}(3)$ [22] have texture similar to the texture II in the present terminology. It was found there that this texture can give non-trivial values of $s_{13}^{2}, s_{23}^{2}$ in several $\Delta\left(6 N^{2}\right)$ groups when symmetry condition (1.2) is used. This does not happen with the antisymmetry condition in case of texture II as argued here. On the other hand, one can obtain correct values for $\theta_{13}$ and $\theta_{23}$ in all the $\Delta\left(3 N^{2}\right)$ groups with texture I when antisymmetry condition is employed. Thus symmetry and antisymmetry conditions appear complementary to each other and allow more possibilities for flavour symmetries $G_{f}$. 


\section{Acknowledgments}

It is pleasure to thank Ketan M. Patel for a careful reading of the manuscript and helpful suggestions. I also thank Department of Science and Technology, Government of India for support under the J.C. Bose National Fellowship programme, grant no. SR/S2/JCB$31 / 2010$.

Open Access. This article is distributed under the terms of the Creative Commons Attribution License (CC-BY 4.0), which permits any use, distribution and reproduction in any medium, provided the original author(s) and source are credited.

\section{References}

[1] C.S. Lam, Determining horizontal symmetry from neutrino mixing, Phys. Rev. Lett. 101 (2008) 121602 [arXiv:0804.2622] [INSPIRE].

[2] C.S. Lam, The unique horizontal symmetry of leptons, Phys. Rev. D 78 (2008) 073015 [arXiv: 0809.1185].

[3] C.S. Lam, A bottom-up analysis of horizontal symmetry, arXiv:0907.2206 [INSPIRE].

[4] C.S. Lam, Group theory and dynamics of neutrino mixing, Phys. Rev. D 83 (2011) 113002 [arXiv: 1104.0055] [INSPIRE].

[5] R. de Adelhart Toorop, F. Feruglio and C. Hagedorn, Discrete flavour symmetries in light of T2K, Phys. Lett. B 703 (2011) 447 [arXiv:1107.3486] [InSPIRE].

[6] R. de Adelhart Toorop, F. Feruglio and C. Hagedorn, Finite modular groups and lepton mixing, Nucl. Phys. B 858 (2012) 437 [arXiv:1112.1340] [INSPIRE].

[7] G. Altarelli, F. Feruglio and L. Merlo, Tri-bimaximal neutrino mixing and discrete flavour symmetries, Fortschr. Phys. 61 (2013) 507 [arXiv:1205.5133] [InSPIRE].

[8] M. Holthausen, K.S. Lim and M. Lindner, Lepton mixing patterns from a scan of finite discrete groups, Phys. Lett. B 721 (2013) 61 [arXiv:1212.2411] [INSPIRE].

[9] B. Hu, Neutrino mixing and discrete symmetries, Phys. Rev. D 87 (2013) 033002 [arXiv:1212.2819] [INSPIRE].

[10] D. Hernandez and A.Y. Smirnov, Lepton mixing and discrete symmetries, Phys. Rev. D 86 (2012) 053014 [arXiv: 1204.0445] [InSPIRE].

[11] D. Hernandez and A.Y. Smirnov, Discrete symmetries and model-independent patterns of lepton mixing, Phys. Rev. D 87 (2013) 053005 [arXiv:1212.2149] [INSPIRE].

[12] M. Holthausen and K.S. Lim, Quark and leptonic mixing patterns from the breakdown of a common discrete flavor symmetry, Phys. Rev. D 88 (2013) 033018 [arXiv:1306.4356] [INSPIRE].

[13] L. Lavoura and P.O. Ludl, Residual $\mathbb{Z}_{2} \times \mathbb{Z}_{2}$ symmetries and lepton mixing, Phys. Lett. B 731 (2014) 331 [arXiv:1401.5036] [INSPIRE].

[14] R.M. Fonseca and W. Grimus, Classification of lepton mixing patterns from finite flavour symmetries, arXiv:1410.4133 [INSPIRE].

[15] B. Hu, Lepton mixing, residual symmetries and trigonometric Diophantine equations, Phys. Rev. D 90 (2014) 073012 [arXiv:1407.4722] [INSPIRE]. 
[16] J. Talbert, [Re]constructing finite flavour groups: horizontal symmetry scans from the bottom-up, JHEP 12 (2014) 058 [arXiv: 1409.7310] [INSPIRE].

[17] G. Altarelli and F. Feruglio, Discrete flavor symmetries and models of neutrino mixing, Rev. Mod. Phys. 82 (2010) 2701 [arXiv: 1002.0211] [InSPIRE].

[18] S.F. King and C. Luhn, Neutrino mass and mixing with discrete symmetry, Rept. Prog. Phys. 76 (2013) 056201 [arXiv:1301.1340] [INSPIRE].

[19] A.Y. Smirnov, Discrete symmetries and models of flavor mixing, J. Phys. Conf. Ser. 335 (2011) 012006 [arXiv:1103.3461] [InSPIRE].

[20] W. Grimus, L. Lavoura and P.O. Ludl, Is $S_{4}$ the horizontal symmetry of tri-bimaximal lepton mixing?, J. Phys. G 36 (2009) 115007 [arXiv:0906.2689] [INSPIRE].

[21] D. Hernandez and A.Y. Smirnov, Relating neutrino masses and mixings by discrete symmetries, Phys. Rev. D 88 (2013) 093007 [arXiv: 1304.7738] [INSPIRE].

[22] A.S. Joshipura and K.M. Patel, Discrete flavor symmetries for degenerate solar neutrino pair and their predictions, Phys. Rev. D 90 (2014) 036005 [arXiv:1405.6106] [INSPIRE].

[23] A.S. Joshipura and K.M. Patel, Horizontal symmetries of leptons with a massless neutrino, Phys. Lett. B 727 (2013) 480 [arXiv:1306.1890] [INSPIRE].

[24] A.S. Joshipura and K.M. Patel, A massless neutrino and lepton mixing patterns from finite discrete subgroups of U(3), JHEP 04 (2014) 009 [arXiv: 1401.6397] [INSPIRE].

[25] G.A. Miller, H.F. Blichfeldt and L.E. Dickson, Theory and applications of finite groups, John Wiley \& Sons, New York U.S.A. (1916).

[26] W.M. Fairbairn, T. Fulton and W.H. Klink, Finite and disconnected subgroups of $\mathrm{SU}_{3}$ and their application to the elementary-particle spectrum, J. Math. Phys. 5 (1964) 1038 [InSPIRE].

[27] A. Bovier, M. Luling and D. Wyler, Finite subgroups of SU(3), J. Math. Phys. 22 (1981) 1543 [INSPIRE].

[28] C. Luhn, S. Nasri and P. Ramond, Simple finite non-abelian flavor groups, J. Math. Phys. 48 (2007) 123519 [arXiv:0709.1447] [InSPIRE].

[29] C. Luhn, S. Nasri and P. Ramond, Flavor group $\Delta\left(3 n^{2}\right)$, J. Math. Phys. 48 (2007) 073501 [hep-th/0701188] [INSPIRE].

[30] J.A. Escobar and C. Luhn, The flavor group $\Delta\left(6 n^{2}\right)$, J. Math. Phys. 50 (2009) 013524 [arXiv: 0809.0639] [INSPIRE].

[31] P.O. Ludl, Systematic analysis of finite family symmetry groups and their application to the lepton sector, arXiv:0907.5587 [INSPIRE].

[32] P.O. Ludl, On the finite subgroups of U(3) of order smaller than 512, J. Phys. A 43 (2010) 395204 [Erratum ibid. A 44 (2011) 139501] [arXiv:1006.1479] [INSPIRE].

[33] R. Zwicky and T. Fischbacher, On discrete minimal flavour violation, Phys. Rev. D 80 (2009) 076009 [arXiv: 0908.4182] [INSPIRE].

[34] K.M. Parattu and A. Wingerter, Tribimaximal mixing from small groups, Phys. Rev. D 84 (2011) 013011 [arXiv: 1012 .2842] [inSPIRE].

[35] W. Grimus and P.O. Ludl, Principal series of finite subgroups of SU(3), J. Phys. A 43 (2010) 445209 [arXiv: 1006. 0098] [INSPIRE]. 
[36] W. Grimus and P.O. Ludl, Finite flavour groups of fermions, J. Phys. A 45 (2012) 233001 [arXiv:1110.6376] [INSPIRE].

[37] W. Grimus and P.O. Ludl, On the characterization of the $\mathrm{SU}(3)$-subgroups of type $C$ and D, J. Phys. A 47 (2014) 075202 [arXiv:1310.3746] [inSPIRE].

[38] A. Merle and R. Zwicky, Explicit and spontaneous breaking of SU(3) into its finite subgroups, JHEP 02 (2012) 128 [arXiv:1110.4891] [INSPIRE].

[39] S.F. King, T. Neder and A.J. Stuart, Lepton mixing predictions from $\Delta\left(6 n^{2}\right)$ family symmetry, Phys. Lett. B 726 (2013) 312 [arXiv:1305.3200] [INSPIRE].

[40] G.-J. Ding, S.F. King and T. Neder, Generalised $C P$ and $\Delta\left(6 n^{2}\right)$ family symmetry in semi-direct models of leptons, JHEP 12 (2014) 007 [arXiv:1409.8005] [INSPIRE].

[41] C. Hagedorn, A. Meroni and E. Molinaro, Lepton mixing from $\Delta\left(3 n^{2}\right)$ and $\Delta\left(6 n^{2}\right)$ and $C P$, Nucl. Phys. B 891 (2015) 499 [arXiv: 1408.7118] [InSPIRE].

[42] H. Ishimori et al., Non-abelian discrete symmetries in particle physics, Prog. Theor. Phys. Suppl. 183 (2010) 1 [arXiv:1003.3552] [InSPIRE].

[43] E. Ma and G. Rajasekaran, Softly broken $A_{4}$ symmetry for nearly degenerate neutrino masses, Phys. Rev. D 64 (2001) 113012 [hep-ph/0106291] [InSPIRE].

[44] K.S. Babu, E. Ma and J.W.F. Valle, Underlying $A_{4}$ symmetry for the neutrino mass matrix and the quark mixing matrix, Phys. Lett. B 552 (2003) 207 [hep-ph/0206292] [INSPIRE].

[45] G. Altarelli and F. Feruglio, Tri-bimaximal neutrino mixing, $A_{4}$ and the modular symmetry, Nucl. Phys. B 741 (2006) 215 [hep-ph/0512103] [InSPIRE].

[46] S. Gupta, A.S. Joshipura and K.M. Patel, Minimal extension of tribimaximal mixing and generalized $Z_{2} \times Z_{2}$ symmetries, Phys. Rev. D 85 (2012) 031903 [arXiv:1112.6113] [INSPIRE].

[47] E. Ma, Transformative $A_{4}$ mixing of neutrinos with CP-violation, Phys. Rev. D 92 (2015) 051301 [arXiv: 1504.02086] [InSPIRE].

[48] X.-G. He, Y.-Y. Keum and R.R. Volkas, $A_{4}$ flavor symmetry breaking scheme for understanding quark and neutrino mixing angles, JHEP 04 (2006) 039 [hep-ph/0601001] [INSPIRE].

[49] G.-N. Li and X.-G. He, CP violation in neutrino mixing with $\delta=-\pi / 2$ in $A_{4}$ type-II seesaw model, Phys. Lett. B 750 (2015) 620 [arXiv:1505.01932] [INSPIRE].

[50] M. Hirsch, A.S. Joshipura, S. Kaneko and J.W.F. Valle, Predictive flavour symmetries of the neutrino mass matrix, Phys. Rev. Lett. 99 (2007) 151802 [hep-ph/0703046] [INSPIRE].

[51] A. Dev, P. Ramadevi and S.U. Sankar, Non-zero $\theta_{13}$ and $\delta_{\mathrm{CP}}$ in a neutrino mass model with $A_{4}$ symmetry, JHEP 11 (2015) 034 [arXiv: 1504.04034] [INSPIRE].

[52] X.-G. He, $A$ model of neutrino mass matrix with $\delta=-\pi / 2$ and $\theta_{23}=\pi / 4$, arXiv: 1504.01560 [INSPIRE].

[53] F. Capozzi et al., Status of three-neutrino oscillation parameters, circa 2013, Phys. Rev. D 89 (2014) 093018 [arXiv: 1312. 2878] [INSPIRE].

[54] D.V. Forero, M. Tortola and J.W.F. Valle, Neutrino oscillations refitted, Phys. Rev. D 90 (2014) 093006 [arXiv: 1405.7540] [INSPIRE]. 
[55] M.C. Gonzalez-Garcia, M. Maltoni and T. Schwetz, Updated fit to three neutrino mixing: status of leptonic CP-violation, JHEP 11 (2014) 052 [arXiv:1409.5439] [INSPIRE].

[56] G. Altarelli and D. Meloni, A simplest A4 model for tri-bimaximal neutrino mixing, J. Phys. G 36 (2009) 085005 [arXiv:0905.0620] [INSPIRE].

[57] S.F. King and C. Luhn, $A_{4}$ models of tri-bimaximal-reactor mixing, JHEP 03 (2012) 036 [arXiv: 1112.1959] [INSPIRE].

[58] S.F. King and C. Luhn, Trimaximal neutrino mixing from vacuum alignment in $A_{4}$ and $S_{4}$ models, JHEP 09 (2011) 042 [arXiv:1107.5332] [INSPIRE].

[59] H.S. Goh, R.N. Mohapatra and S.-P. Ng, Testing neutrino mass matrices with approximate $L_{e}-L_{\mu}-L_{\tau}$ symmetry, Phys. Lett. B 542 (2002) 116 [hep-ph/0205131] [INSPIRE].

[60] W. Grimus, S. Kaneko, L. Lavoura, H. Sawanaka and M. Tanimoto, $\mu-\tau$ antisymmetry and neutrino mass matrices, JHEP 01 (2006) 110 [hep-ph/0510326] [INSPIRE]. 